\title{
Determination of the shape and indium distribution of low-growth-rate InAs quantum dots by cross-sectional scanning tunneling microscopy
}

\author{
D. M. Bruls, ${ }^{\text {a) }}$ J. W. A. M. Vugs, P. M. Koenraad, H. W. M. Salemink and J. H. Wolter \\ COBRA Inter-University Research Institute, Dept. of Applied Physics, Eindhoven University of Technology, \\ P.O. Box 513, 5600 MB Eindhoven, The Netherlands \\ M. Hopkinson \\ Department of Electronic and Electrical Engineering, University of Sheffield, Mappin Street, Sheffield S1 3JD, \\ United Kingdom
}

M. S. Skolnick

Department of Physics and Astronomy, University of Sheffield, Sheffield S3 7RH, United Kingdom

Fei Long and S. P. A. Gill

Department of Engineering, University of Leicester, Leicester LE1 7RH, United Kingdom

(Received 27 August 2001; accepted for publication 10 July 2002)

\begin{abstract}
We present a cross-sectional scanning-tunneling microscopy investigation of the shape, size, and composition of InAs quantum dots in a GaAs matrix, grown by molecular beam epitaxy at low growth rate. From the dimensional analysis we conclude that the investigated quantum dots have an average height of $5 \mathrm{~nm}$, a square base of $18 \mathrm{~nm}$ oriented along [010] and [100] and the shape of a truncated pyramid. From outward relaxation and lattice constant profiles we conclude that the dots consist of an InGaAs alloy and that the indium concentration increases linearly in the growth direction. Our results justify the predictions obtained from previous photocurrent measurements on similar structures and the used theoretical model. (C) 2002 American Institute of Physics.
\end{abstract}

[DOI: $10.1063 / 1.1504162]$

The shape and composition of self-assembled quantum dots (SQDs) are properties that are very difficult to determine and often only available by indirect means. Precise knowledge of these parameters, ${ }^{1-7}$ on which optical and electrical properties depend, are of major interest. InAs SQDs in a GaAs matrix, grown by molecular beam epitaxy (MBE) in the Stranski-Krastanov growth mode, are of particular interest. Quantum dots at the growth surface can be investigated using atomic force microscopy. ${ }^{8}$ The dot shape and thus the electronic properties can, however, change significantly after coverage with a capping layer. High resolution transmission electron microscopy can be used to investigate covered quantum dots, ${ }^{1,6,7,9,10}$ but this method suffers from averaging effects, which hamper the investigation of, for example, interface roughness and alloy fluctuations. In this study, cross-sectional scanning-tunneling microscopy (X-STM) is employed to obtain a conclusive and comprehensive determination of the shape, size, and composition of SQDs. These results are in good agreement with photocurrent measurements made previously on a similar structure, ${ }^{1}$ and justify the theoretical assumptions that were used to fit these photocurrent measurements.

The X-STM measurements were performed under UHV $\left(\mathrm{p}<4.10^{-11}\right.$ Torr) conditions, using an Omicron STM1, TS-2 scanner, on in-situ cleaved (110) surfaces. All structures were grown by $\mathrm{MBE}$ at $512{ }^{\circ} \mathrm{C}$ and contained five layers of low growth rate $(0.01$ monolayers $(\mathrm{ML}) / \mathrm{s}, 2.4 \mathrm{ML}$ InAs per layer deposited) InAs dots within a GaAs matrix. The dots were located in the middle of a $p-i-n$ junction to

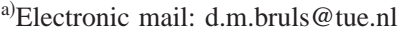

enable bias dependent photocurrent measurements. ${ }^{1}$ The dot layers are uncoupled with a spacing of $50 \mathrm{~nm}$ between the layers.

A number of individual SQDs were investigated by X-STM using the constant current mode in which both the topography and current image were recorded. In Fig. 1 we display a typical current image of a single investigated dot

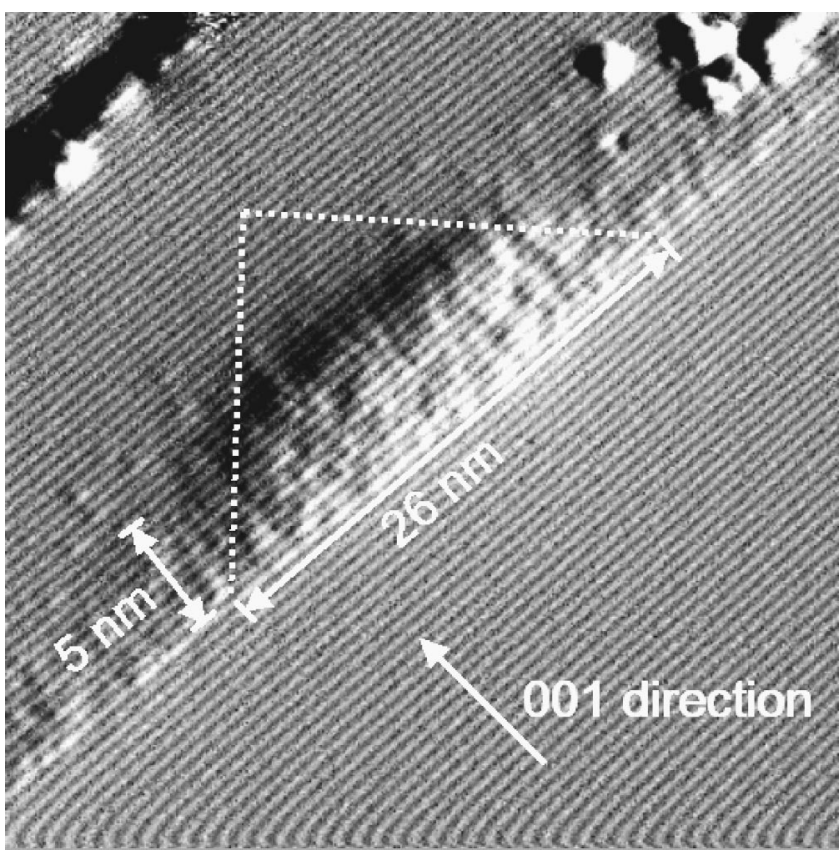

FIG. $1.40 \times 40 \mathrm{~nm}^{2} \mathrm{X}$-STM current image of a cleaved InAs quantum dot and the wetting layer. In the upper right corner some cleavage debris is visible. 
1

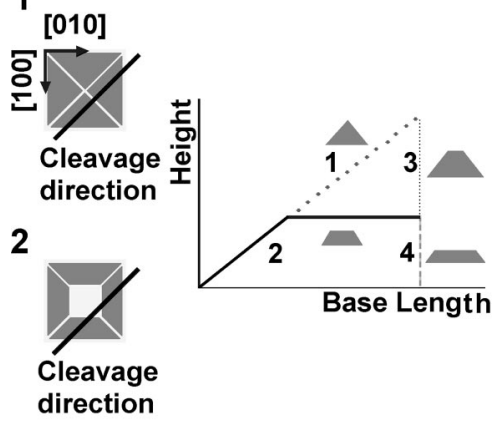

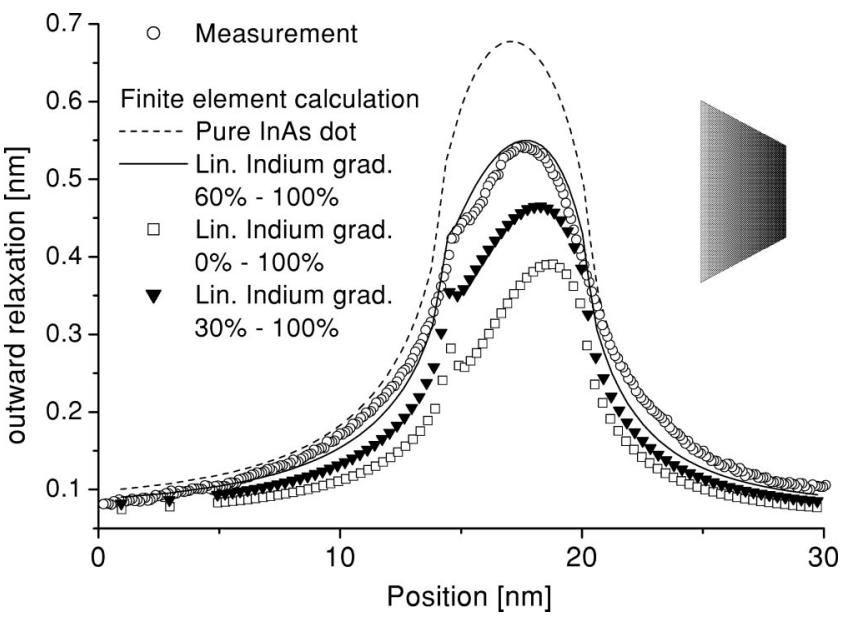

FIG. 4. Measured and calculated line profiles across the center of the dot. All indium gradients and the measured profile are plotted from bottom to top.

the square base, so the actual base length is $18 \pm 1 \mathrm{~nm}$. The height of the dots is $5 \pm 1 \mathrm{~nm}$. This is in perfect agreement with photocurrent measurements performed previously on a sample grown under very similar conditions. ${ }^{1,2}$ Moreover, they concluded that an indium gradient must exist in these SQDs. In the measured quantum dots, many small short ranged fluctuations are visible (Fig. 1) indicating that the dot material is indeed an alloy. The formation of an alloy during growth is well known from STM measurements on uncapped InAs quantum dot structures. ${ }^{3}$

After cleavage the quantum dot relaxes outwards due to the strain resulting from the large lattice mismatch between InAs and GaAs (7\%) (Fig. 4). This is visible in line profiles across the dots in topography images. The magnitude of this outward relaxation is linked to the local indium concentration inside the quantum dot $^{11}$ (Fig. 4). By using the proper tunnel conditions, only the true surface relaxation is imaged and the electronic effects in the height corrugation due to band gap differences are negligible. ${ }^{14}$

Using the finite element calculation package ABAQUS, which was successfully used for the interpretation of photocurrent measurements, ${ }^{1}$ it is possible to calculate the outward relaxation (Fig. 4) and lattice constant (Fig. 5) profiles for cleaved dots with finite dimensions consisting of various indium distributions, like a linear indium gradient. In the calculations, different values for Young's modulus ( $E_{\text {InAs }}$ $\left.=51.44 \mathrm{GPa}, E_{\mathrm{GaAs}}=85.62 \mathrm{GPa}\right)$ and Poisson's ratio $\left(\nu_{\mathrm{InAs}}\right.$ $\left.=0.353, \nu_{\mathrm{GaAs}}=0.3177\right)$ are assumed inside and outside the dot, respectively. Furthermore, these parameters and the strain in the alloy are assumed to be a linear function of the indium concentration. Although some particular shape and composition combinations could result in the same relaxation profile, these calculations, together with the X-STM measurements, can be very effectively used to determine the local indium concentration in the SQDs within $10 \%$.

The calculations have been performed for various compositions for a dot with the shape of a square based truncated pyramid and have been compared with experimental results (Figs. 4 and 5). The width of the dot at the bottom is $18 \mathrm{~nm}$ and decreases to $10.6 \mathrm{~nm}$ at the top of the dot. The dot height is $5.0 \mathrm{~nm}$, it sits on a 0.6 -nm-thick continuous wetting layer

FIG. 3. Height versus base length distribution of the cross-sectional images of the analyzed quantum dots. The expected distributions for the two possible cross-sections with the $\{111\}$ (dotted line) and $\{101\}$ (solid line) facets, forming the sidewalls of the dots, are indicated in the graph. 


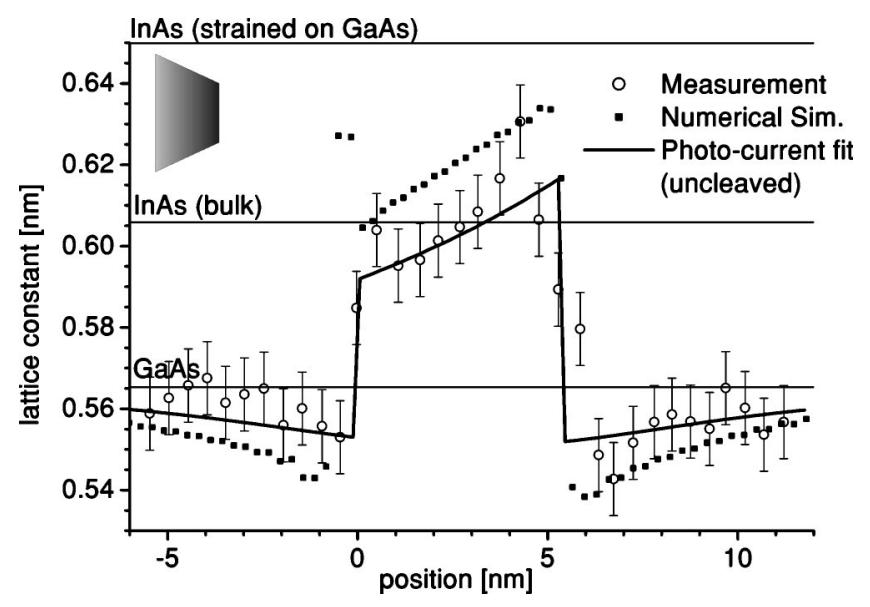

FIG. 5. Average measured lattice constant profile in the growth direction of the quantum dot (circle), compared with calculated profiles from the photocurrent results (uncleaved surface) (solid line) and numerical simulations (square).

and is assumed to be cleaved along its diagonal. In the calculations the following indium profiles in de dots are investigated: (1) $\operatorname{In}_{x} \mathrm{Ga}_{1-x}$ As dots with constant $x$, (2) dots with a linear indium gradient, (3) dots with a pure inverted pyramidal shaped indium core, and (4) dots with indium profiles as proposed in Ref. 15.

By analyzing only dots with a cross-sectional base length that equals the maximum observed value (Fig. 3), we are sure that the experimental data presented in Figs. 4 and 5 are indeed obtained from dots that are cleaved along their diagonal. Calculations assuming steeper side facets of the dot, which are not observed, result in a worse outward relaxation fit. A lower indium concentration indeed results in a lower average outward relaxation, and the dots cannot consist of pure InAs due to the asymmetry in the profile. The calculation assuming an indium gradient (60\% linear to $100 \%$ from bottom to top) yields the best fit to our relaxation measurements (Fig. 4). In addition, we find a reasonable agreement for the same parameter set for the lattice constant profile in Fig. 5, where we have determined the change in lattice constant throughout the cleaved dot by taking line profiles and measuring the spacing between the atom rows.

We observe an increase of the lattice constant of $35 \mathrm{pm}$ going from the bottom to the top of the cleaved dot. This can be linked to the local indium content. ${ }^{11}$ In principle a variation of the lattice constant in the dots does not imply a gradient in the indium concentration. By using the finite element calculations we were able to show that from the investigated models, only the models assuming an indium gradient towards the top of the dot, as proposed in Refs. 1 and 2, and which gave the best fit to the measured relaxation profiles, can give rise to the observed increased strain in the growth direction, causing the observed lattice constant profile of the cleaved dot (Fig. 5). The indium gradient is possible through growth processes such as segregation and strain related indium incorporation. ${ }^{9,16}$ Both these mechanisms might be expected to lead to an indium gradient, resulting in a higher indium concentration in the top of the dot.

In conclusion, we show that X-STM can be used very effectively to determine shape, size, and local indium concentration of SQDs in a quantitative way. From outward relaxation, lattice constant profiles, and numerical calculations we have concluded that the indium concentration within the dots, which were grown at low growth rate, increases linearly towards the top. This is in agreement with predictions of previous photocurrent measurements, justifying the theoretical approach used in Ref. 1. Therefore the application of $\mathrm{X}$-STM, together with theoretical modeling, can give a correct description of the optoelectronic properties of SQDs.

The authors would like to acknowledge J. A. Barker and E. P. O'Reilly for making the data of the strain distribution, used in Ref. 2 available to them, J. H. Davies and Prof. A. Cocks for fruitful discussions and the EPSRC for funding (Grant No. GR/M31705).

${ }^{1}$ P. W. Fry, I. E. Itskevich, D. J. Mowbray, M. S. Skolnick, J. J. Finley, J. A. Barker, E. P. O'Reilly, L. R. Wilson, I. A. Larkin, P. A. Maksym, M. Hopkinson, M. Al-Khafaji, J. P. R. David, A. G. Cullis, G. Hill, and J. C. Clark, Phys. Rev. Lett. 84, 733 (2000).

${ }^{2}$ J. A. Barker and E. P. O'Reilly, Phys. Rev. B 61, 13840 (2000).

${ }^{3}$ P. B. Joyce, T. J. Krzyzewski, G. R. Bell, B. A. Joyce, and T. S. Jones, Phys. Rev. B 58, 15981 (1998).

${ }^{4}$ I. Kegel, T. H. Metzger, A. Lorke, J. Peisl, J. Stangl, G. Bauer, J. M. García, and P. M. Petroff, Phys. Rev. Lett. 85, 1694 (2000).

${ }^{5}$ B. Grandidier, Y. M. Niquet, B. Legrand, J. P. Nys, C. Priester, D. Stiévenard, J. M. Gérard, and V. Thierry-Mieg, Phys. Rev. Lett. 85, 1068 (2000).

${ }^{6}$ D. Zhi, H. Davock, R. Murray, C. Roberts, T. S. Jones, D. W. Pashley, P. J. Goodhew, and B. A. Joyce, J. Appl. Phys. 89, 2079 (2001).

${ }^{7}$ A. Rosenauer, W. Oberst, D. Litvinov, D. Gerthsen, A. Förster, and R. Schmidt, Phys. Rev. B 61, 8276 (2000).

${ }^{8}$ D. Leonard, K. Pond, and P. M. Petroff, Phys. Rev. B 50, 11687 (1994).

${ }^{9}$ Q. Xie, A. A. Madhukar, P. Chen, and N. P. Kobayashi, Phys. Rev. Lett. 75, 2542 (1995).

${ }^{10}$ J. P. McCaffrey, M. D Robertson, S. Fafard, Z. R. Wasilewski, E. M. Griswold, and L. D. Madsen, J. Appl. Phys. 88, 2272 (2000).

${ }^{11}$ O. Flebbe, H. Eisele, T. Kalka, F. Heinrichsdorff, A. Krost, D. Bimberg, and M. Dähne-Prietsch, J. Vac. Sci. Technol. B 17, 1639 (1999).

${ }^{12}$ V. A. Shchukin and D. Bimberg, Rev. Mod. Phys. 71, 1125 (1999).

${ }^{13}$ I. Daruka, J. Tersoff, and A.-L. Barabási, Phys. Rev. Lett. 82, 2753 (1999).

${ }^{14}$ D. M. Bruls, P. M. Koenraad, M. Hopkinson, J. H. Wolter, and H. W. M. Salemink, Appl. Surf. Sci. 190, 258 (2002).

${ }^{15}$ N. Liu, J. Tersoff, O. Baklenov, A. L. Holmes, Jr., and C. K. Shih, Phys. Rev. Lett. 84, 334 (2000).

${ }^{16}$ M. Pfister, M. B. Johnson, S. F. Alvarado, and H. W. M. Salemink, Appl. Phys. Lett. 67, 1459 (1995). 